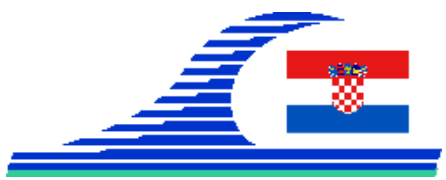

\title{
Evolution morphodynamique du trait de côte à Saïdia et à Al Hoceima sur le littoral méditerranéen Marocain
}

\author{
Khadija AIT LEMQEDDEM ${ }^{1}$, Hajer BENJILANI ${ }^{1}$, Brahim DAMNATI ${ }^{1}$
}

1. Université Abdelmalek Essaadi, Faculté des Sciences et Techniques de Tanger, Département des Sciences de la Terre, Équipe Environnement Marin et Risques Naturels, B.P 416, Tanger Principal, 90000 Maroc.

khadijaait.lemqeddem@gmail.com

\section{Résumé :}

La présente étude traite l'évolution du trait de côte, en évaluant les risques d'érosion et l'impact des changements climatiques sur le littoral de Saïdia et d'Al Hoceima au Nord du Maroc durant les dernières décennies. Ainsi, la méthodologie de la photointerprétation a été appliquée.

L'étude cartographique, basée sur l'analyse multi date des images satellitaires sur 7 ans, au niveau de Saïdia, a permis de confirmer un recul du trait de côte. Le taux moyen annuel d'érosion côtière varie entre $-0,58 \mathrm{~m} / \mathrm{an}$ et $-5,70 \mathrm{~m} / \mathrm{an}$. Une partie de cette zone était relativement stable entre 1958 et 2006.

Au niveau de la zone d'étude d'Al Hoceima, cette analyse sur 12 ans a montré un taux d'érosion moyen de $-2 \mathrm{~m} / \mathrm{an}$ à l'Ouest de la plage Sfiha. Dans la partie Est, cette érosion est relativement faible.

Mots clés : Erosion côtière, Trait de côte, Multi date, Risque d'inondation, Al Hoceima, Saïdia, Maroc, Méditerranée.

\section{Introduction}

La zone d'étude se situe sur la frange côtière Méditerranéenne du Maroc. Elle comprend la côte Rifaine et la côte Nord Orientale (DAKKI, 2004). Elle se caractérise par un climat semi-aride à humide, à influence méditerranéenne, avec des précipitations qui ne dépassent pas $600 \mathrm{~mm}$ par an, des étés chauds et secs et des hivers frais et humides. De ce fait, les écosystèmes côtiers et marins sont extrêmement diversifiés et abritent une variété d'espèces et des arbres forestiers. La marée est de faible amplitude en méditerranée et son gradient diminue vers l'est. Elle est la conséquence de la marée atlantique. Cette zone est riche par son immense patrimoine naturel, culturel et économique. Cependant, ce patrimoine est soumis à de très fortes pressions dont la dégradation de la qualité des eaux, la faible gestion des déchets solides des décharges publiques, le risque d'inondation, une biodiversité menacée, la déforestation et l'érosion des plages. 
Mediterranean rocky coasts:

Features, processes, evolution and problems

L'aire de cette étude se situe sur la frange côtière méditerranéenne du Maroc et sur deux zones. La côte d'Al Hoceima s'étend sur une longueur de 1650 m environ et une largeur de $50 \mathrm{~m}$. Elle est située dans la partie centrale au Nord du Maroc. Le littoral de Saïdia s'étend sur une longueur de $14 \mathrm{~km}$ et une largeur de $250 \mathrm{~m}$. Il est situé au Nord-Est du Maroc. Cette dernière zone est bordée à l'Ouest par Ras Elma et à l'extrémité Est par l'oued Kiss qui marque la frontière entre le Maroc et l'Algérie (figure 1).
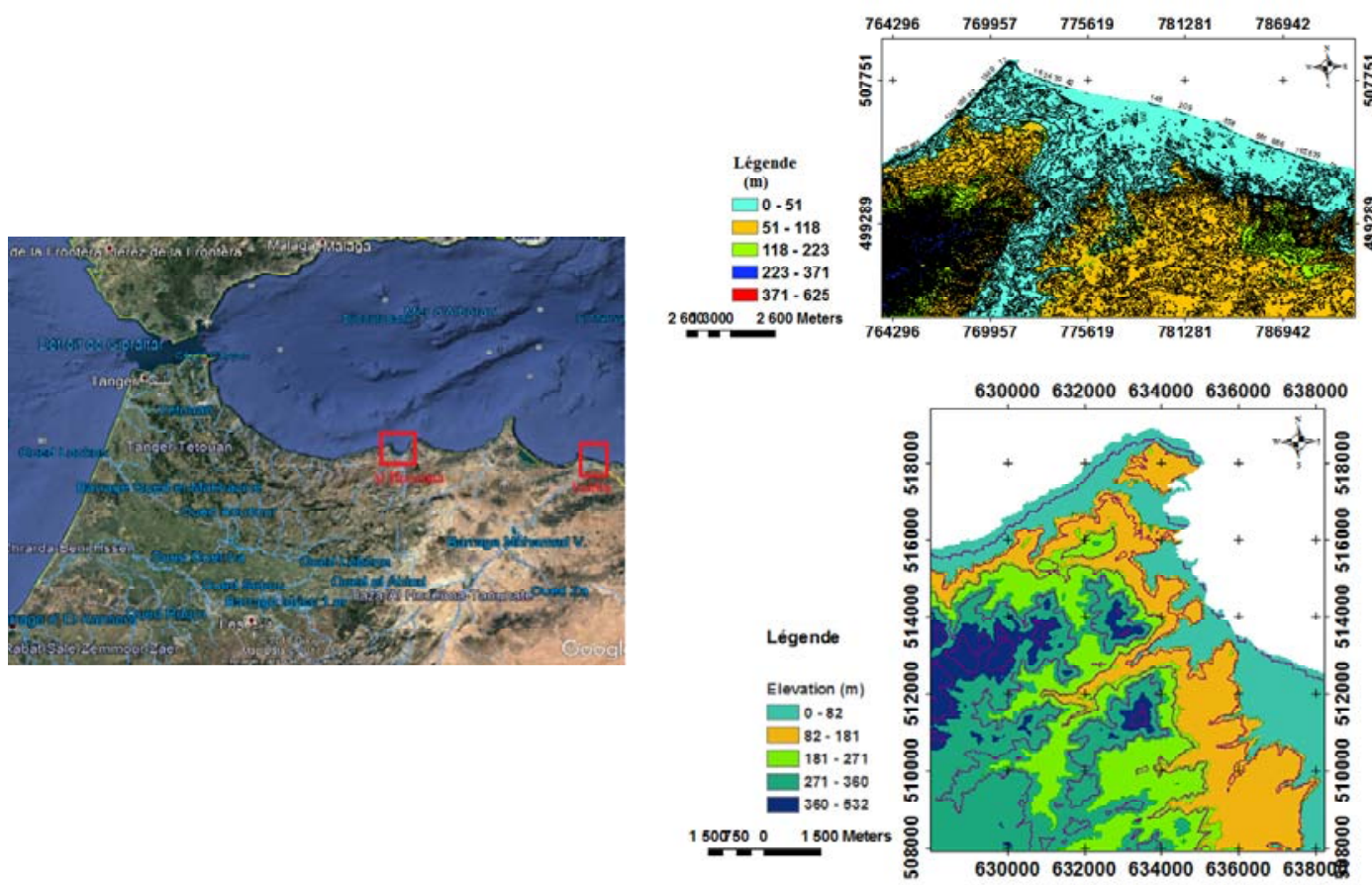

Figure 1. Carte de localisation de la zone d'étude de Saïdia (en haut) et d'Al Hoceima (en bas) sur la côte méditerranéenne Marocaine.

\section{Méthodologie}

L'évolution du trait de côte de Saïdia et d'Al Hoceima, a été reconstituée à partir d'une approche cartographique. Elle est basée sur l'analyse multi date des images satellitaires verticales et des cartes topographiques comme document de référence pour servir de base à la correction. Les cartes de Triffa-Saidia à l'échelle de 1/50 000 et d'Al Hoceima à l'échelle 1/100 000 ont été utilisées. Pour suivre la mobilité de la ligne de rivage, un traitement a été fait à l'aide de l'outil système d'information géographique en utilisant de logiciel Arc GIS 10.1 (EL ABDELLAOUI \& OZER, 2007; BOUABDALLAH \& LARUE, 2009). Les taux d'érosion et ou d'engraissement, le calcul de la distance et la superficie entre deux traits de côte, ont été estimés entre deux images multi date.

Les différentes classes qui ont été définies sont :

- Erosion : forte ( $>-3 \mathrm{~m} / \mathrm{an})$, faible (<-2 m/an) et stable $(<-0,20 \mathrm{~m} / \mathrm{an})$. 
Mediterranean rocky coasts:

Features, processes, evolution and problems

- Engraissement : fort $(>+10 \mathrm{~m} / \mathrm{an})$, faible ( $<+2 \mathrm{~m} / \mathrm{an})$ et stable $(<+1 \mathrm{~m} / \mathrm{an})$.

\section{Résultats et discussion}

L'analyse de l'évolution du trait de côte au niveau d'Al Hoceima montre d'importants changements entre 2004 et 2016 (figure 2). La zone Ouest de la plage, montre un fort taux d'érosion de (-2,08 m/an). Ce taux peut être expliqué d'une part, par les conditions climatiques exceptionnelles au cours de l'année 2012, avec de fortes précipitations et de fortes tempêtes (DAMNATI \& AIT LEMQEDDEM, 2015). D'autre part, il peut être expliqué par la dominance des vents Est-Nord-Est le long de la côte et par l'action des courants de houle de direction Ouest-Nord-Ouest. La zone à l'extrême Est, est considérée comme une zone de dépôts des sédiments transportés par les vents. Le taux d'érosion est faible par rapport à la zone Ouest $(-1,28 \mathrm{~m} / \mathrm{an})$ malgré le fort hydrodynamisme. Ceci est lié à l'existence d'un couvert végétal dense et la présence d'une île (ile Nekkor) en face des trajectoires des courants de houles (Ouest-NordOuest) qui protègent cette dernière zone. Globalement, 41 \% de cette zone subit une forte érosion et $55 \%$ subit une faible érosion (figure 2).

Dans les prochaines années le volume moyen de l'érosion à Al Hoceima serait de plus de $3375 \mathrm{~m}^{3}$. Ceci va affecter directement et/ou indirectement l'écosystème littoral et beaucoup d'infrastructures de cette région.

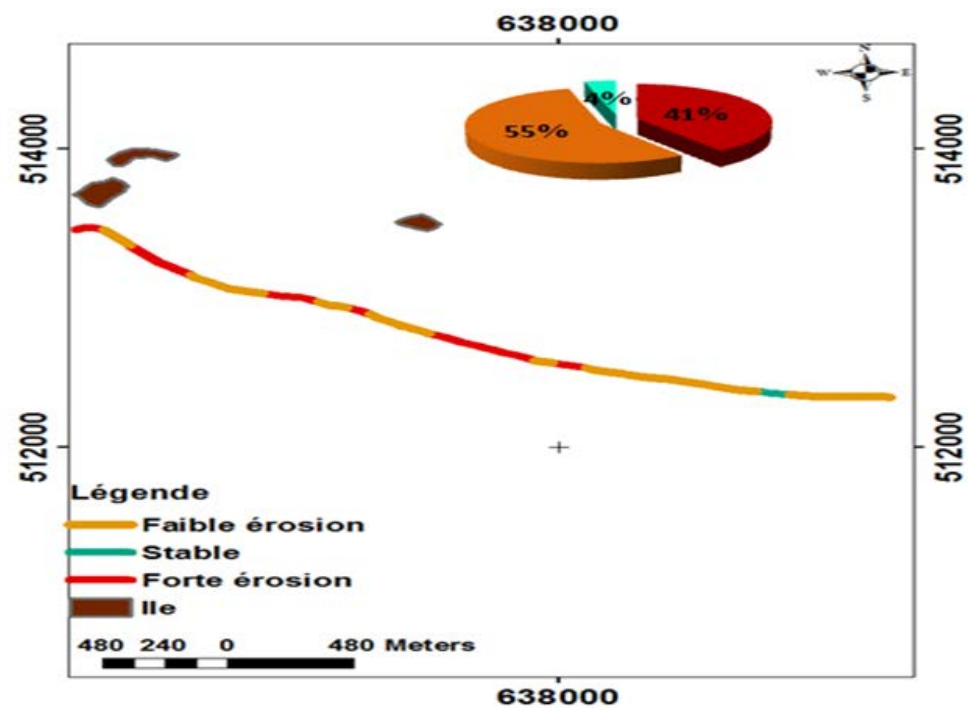

Figure 2. Carte des risques d'érosion au niveau d'Al Hoceima pendant la période 2004-2016.

Au niveau de la deuxième zone d'étude de Saïdia (figure 3), le trait de côte est généralement en recul. Plus de $48 \%$ de cette zone est en érosion et $22 \%$ est en 
Mediterranean rocky coasts:

Features, processes, evolution and problems

engraissement. Le taux moyen annuel d'érosion côtière varie entre $-0,58 \mathrm{~m} / \mathrm{an}$ et $-5,70$ $\mathrm{m} / \mathrm{an}$.

A l'Est de cette zone, la plage est en érosion avec des taux moyens variant entre -1,18 $\mathrm{m} / \mathrm{an}$ et $-2,45 \mathrm{~m} / \mathrm{an}$. La zone Est de l'Oued Moulouya est la zone la plus touchée avec un fort taux d'érosion -5,70 m/an. Deux raisons peuvent expliquer ceci, d'abord le fort hydrodynamisme avec des courants d'Est et de Nord-Est ; et aussi la forte tempête qu'a connu la région en 2009 (DAMNATI \& AIT LEMQEDDEM, 2015). La partie à l'extrême Est, marquée par un faible hydrodynamisme, est en engraissement avec un taux de $+13,05 \mathrm{~m} / \mathrm{an}$. Cette même zone était relativement stable entre 1958 et 2006 (BOUABDALLAH \& LARUE, 2009; MOUZOURI \& IRZI, 2011). Dans les prochaines années, le volume moyen de l'érosion côtière à Saïdia serait de plus de $8035 \mathrm{~m}^{3}$.

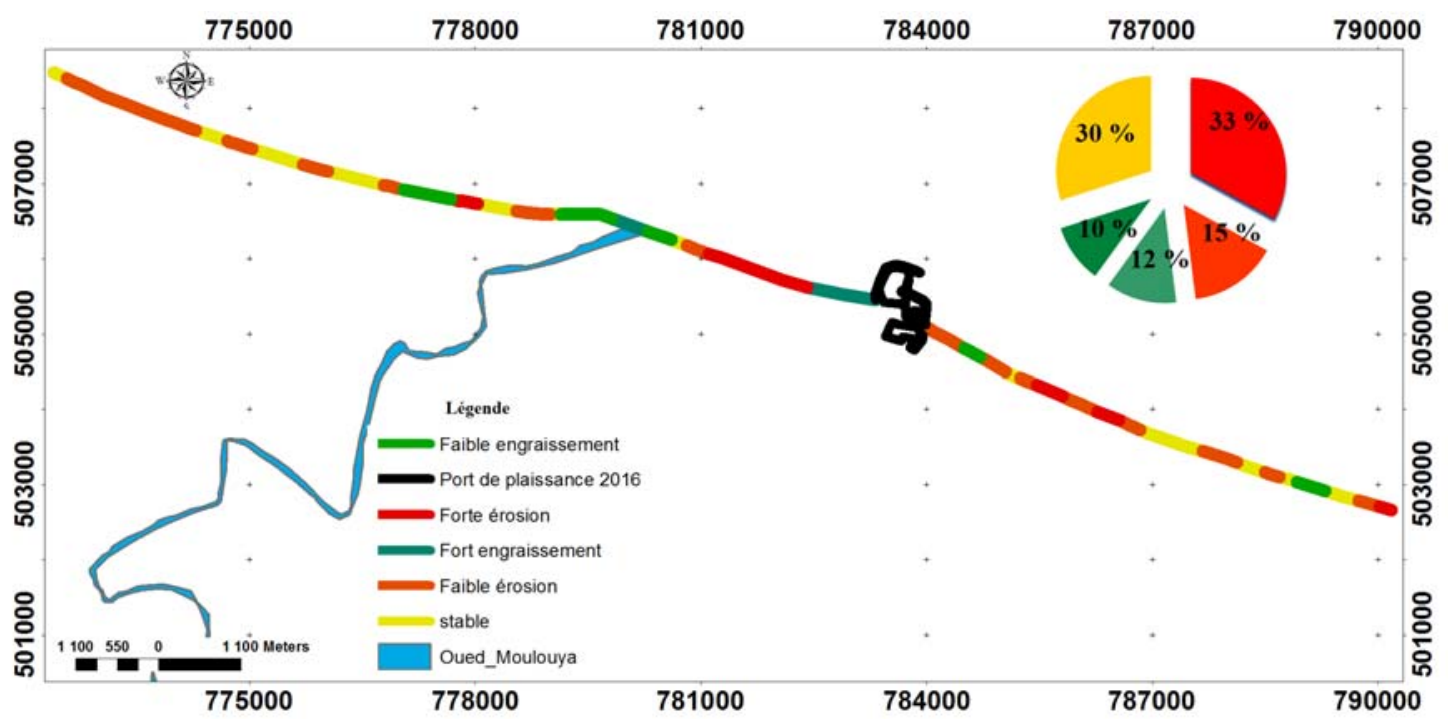

Figure 3. Carte des risques d'érosion du littoral de Saïdia pendant la période 20092016.

\section{Conclusion}

L'analyse de l'évolution du trait de côte au niveau d'Al Hoceima, et Saïdia montre d'importants changements entre 2004 et 2016. A Al Hoceima, la zone Ouest de la plage, présente un taux d'érosion relativement élevé. La zone Est, est considérée comme une zone de dépôts et le taux d'érosion y est faible par rapport à la zone Ouest, malgré le fort hydrodynamisme. A l'Est de Saïdia, la plage est en érosion, surtout au niveau de la partie Est de l'Oued Moulouya. Le volume moyen global de l'érosion dans les deux régions varierait entre 3300 et $8000 \mathrm{~m}^{3}$. Ceci va affecter directement et/ou indirectement l'écosystème littoral et beaucoup d'infrastructures de ces deux régions surtout dans le cadre d'une perspective d'un fort impact des futurs changements climatiques. 
Mediterranean rocky coasts:

Features, processes, evolution and problems

\section{Références bibliographiques}

BOUABDALLAH M., LARUE J.P. (2009). Evolution du littoral de la baie de Saïdia: Dynamique naturelle et impact des aménagements (Maroc Oriental). Revue PhysioGéo, Vol. 3, pp.113-130.

DAKKI M. (2004). Programme d'aménagement côtier en Méditerranée Marocaine: Etude de faisabilité. Direction du Plan d'Action pour la Méditerranée (PAM), Ministère de l'Aménagement du Territoire, de l'Eau et de l'Environnement, Département de l'Environnement, 113 p.

DAMNATI B., AIT LEMQEDDEM K. (2015). Impact des changements climatiques sur le littoral Méditerranéen : exemple du littoral de Saïdia et de Tanger. Recueil des résumés, $22^{\text {ème }}$ colloque international des bassins sédimentaires, pp. 215-218.

EL ABDELLAOUI J.E., OZER A. (2007). Etude diachronique et historique de l'évolution du trait de côte de la baie de Tanger (Maroc). Revue Télédétection, Vol. 7, pp. 157-171.

MOUZOURI M., IRZI Z. (2011). Évolution et morpho-dynamique de la plaine côtière de Saïdia: littoral méditerranéen du Nord-Est du Maroc durant la période 1958-2006. Bulletin de l’Institut Scientifique, Rabat, section Sciences de la Terre, n³3, pp. 65-76. 
Mediterranean rocky coasts:

Features, processes, evolution and problems 